\title{
A Framework for Drawing Planar Graphs with Curves and Polylines
}

\author{
Michael T. Goodrich ${ }^{1 \star}$ and Christopher G. Wagner ${ }^{2 \star \star}$ \\ 1 The Johns Hopkins University goodrich@cs.jhu.edu \\ 2 The Johns Hopkins University wagner@mts.jhu.edu \\ http://www.mts.jhu.edu/ wagner
}

\begin{abstract}
We describe a unified framework of aesthetic criteria and complexity measures for drawing planar graphs with polylines and curves. This framework includes several visual properties of such drawings, including aspect ratio, vertex resolution, edge length, edge separation, and edge curvature, as well as complexity measures such as vertex and edge representational complexity and the area of the drawing. In addition to this general framework, we present algorithms that operate within this framework. Specifically, we describe an algorithm for drawing any $n$ vertex planar graph in an $O(n) \times O(n)$ grid using polylines that have at most two bends per edge and asymptotically-optimal worst-case angular resolution. More significantly, we show how to adapt this algorithm to draw any $n$-vertex planar graph using cubic Bézier curves, with all vertices and control points placed within an $O(n) \times O(n)$ integer grid so that the curved edges achieve a curvilinear analogue of good angular resolution. All of our algorithms run in $O(n)$ time.
\end{abstract}

\section{Introduction}

One of the main contributions of research in graph drawing has been the formal identification of aesthetic criteria that graph drawings can possess together with the trade-offs that exist between these criteria and various complexity measures of drawings. Such formal specifications allow us to quantify the qualities that make a drawing "nice" to look at and studies of various trade-offs further our understanding of which aesthetic goals can be realistically achieved. Examples of such studies are too numerous to enumerate, but some well-known examples include the importance of the drawing area identified by de Fraysseix, Pach, and Pollack [3], the illumination of the value of bend minimization in polyline drawings by Tamassia [13, the discussion of aspect ratio by Chan et al. [1], and the identification of angular resolution as a signficant aesthetic criterion by Formann et al. [5], and its further study by Kant [10, Garg and Tamassia [7, and Malitz and Papakostas [11].

\footnotetext{
* Work by this author is supported in part by the U.S. Army Research Office under Grant DAAH04-96-1-0013 and by NSF under Grant CCR-96-25289.

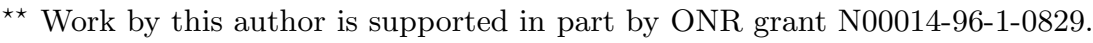


This paper is directed at continuing this tradition by articulating a unified framework for describing aesthetic criteria and complexity measures for drawings of graphs that place vertices at points in the plane and represent edges with smooth curves (that is, curves with at least $\mathcal{C}^{1}$ continuity) or polylines (that is, polygonal chains). We review or generalize several well-known graph-drawing design goals for this framework, including aspect ratio, vertex resolution, and bend minimization, as well as introduce design goals that are not as well-known, such as a curvilinear analogues to angular resolution and edge resolution. In addition to articulating this framework for curve and polyline drawings, we also describe new planar graph drawing algorithms that operate within this framework.

\subsection{Related Prior Work}

Before we describe our framework and our algorithms for this framework, however, let us review some related prior work. Specifically, since we reviewed above much of the prior work on the identification and study of general aesthetic criteria, let us review here some of the prior work relating to the drawing of planar graphs with curves and polylines.

One of the now-classic results for drawing planar graphs is an algorithm by de Fraysseix, Pach, and Pollack [3] for drawing an $n$-vertex planar graph in an $O(n) \times O(n)$ integer grid using straight line segments to represent edges. Originally presented as an $O(n \log n)$ time algorithm, Chrobak and Payne [2] show how to cleverly use a tree data structure to reduce the running time of this algorithm to $O(n)$. This algorithm does not achieve good angular resolution, however, because edges incident on the same vertex are not necessarily nicely "spread out." But this algorithm introduces an important concept known as the canonical ordering of the vertices of a plane graph, which is vital to several other graph drawing algorithms, including several algorithms by Kant [10]. In particular, Kant uses this ordering in an algorithm to draw a planar graph with vertices placed in an $O(n) \times O(n)$ integer grid and edges drawn as polygonal chains with at most three bends per edge in order to obtain good angular resolution. Forshadowing the topic of our paper, Kant observes that polyline drawings can be modified to give drawings with nicely curved edges, by, say, using splines.

Unfortunately, several issues related to curve drawings are not addressed by Kant. For example, if one applies standard methods for "smoothing" polylines, there may be no guarantee that edges that previously did not cross will not cross in their smoothed form. In addition, there are several complexity issues related to polyline smoothing that should be addressed, such as deciding if a polyline with three bends is better represented using a single degree- 4 curve or as two degree-3 curves joined at a point (which is called a knot). Equally important is the impact that such a decision has on the beauty of the drawing. Indeed, questions such as this are the driving force behind our search for a unified framework for characterizing graph drawings that use polylines and/or curves to represent edges.

On the topic of curve drawings themselves much less is known. There are several systems (including most general drawing packages) that allow for curves 
and polyline smoothing, but we have not been able to find much written on formal studies of curve drawings. A notable exception to this is work of Gansner, Koutsofios, North, and Vo [6] that addresses the issue of curve drawings for general graphs, considering aesthetic criteria such as the hierarchical structure of the underlying graph, the edge length, symmetry, and the number of edge crossings. Their analysis is not as formal as, say, the work of Kant 10] for polyline drawings, but Gansner et al. nevertheless provide evidence that their algorithm runs quickly and produces drawings that compare favorably with previous work. Unfortunately, the heuristics upon which their algorithm is based do not easily provide concrete statements about the properties of the drawings produced. For example, it seems that a drawing of a planar graph produced by this algorithm may have edge crossings. Thus, there is a need for algorithms for drawing graphs with curved edges so as to guarantee various aesthetic and complexity goals.

\subsection{Our Results}

In this paper we review and generalize several known aesthetic and complexity goals to a unified framework for drawing graphs with polyline and/or curve edges. Some of the novel aspects of this framework include a design goal we call edge separation, which is a curvilinear analogue to the concepts of edge resolution and angular resolution used for polyine drawings, as well as a concept we call curvature minimization, which provides a curvilinear analogue to the avoidance of sharp bends in polyline drawings.

In addition to the presentation of this framework, we provide algorithms that operate within it and for which we can make concrete performance claims. Specifically, we give an algorithm to draw an $n$-vertex planar graph in $O(n)$ time in an $O(n) \times O(n)$ integer grid using polyline edges that have at most two bends per edge and achieve an asymptotically-optimal worst-case angular resolution. Thus, we are able to achieve similar goals to a polyline drawing of Kant [10], but with only two bends per edge instead of three. More importantly, by limiting each polyline to two bends, we show how to extend our algorithm to draw an $n$-vertex planar graph in $O(n)$ time in an $O(n) \times O(n)$ grid so that each edge is drawn as a single cubic Bézier curve (i.e., with no knots) in a way that achieves good edge separation and has every vertex and control point placed at points with integer coordinates. Before we give the details of these algorithms, however, let us describe our unified framework for drawing graphs with polylines and curves.

\section{A Framework for Drawing with Curves and Polylines}

The goal of many graph drawing algorithms is to visually represent the essential properties of the graph in an intuitive and pleasing drawing. 


\subsection{Aesthetic Criteria}

Based on this primary objective, we consider several formal aesthetic criteria for drawing graphs with edges represented as polylines and/or curves. Most of these criteria are not new, but are instead either direct translations of known aesthetic criteria to this combined curve/polyline framework or are generalizations of known polyline aesthetic criteria so that they also apply to curve drawings. In particular, we consider the following aesthetic criteria:

Aspect Ratio: If we consider the smallest rectangle that encloses a drawing, the aspect ratio is the ratio of the longer side of the rectangle to the shorter. In most cases, aspect ratios closer to 1 are more desirable.

Vertex Resolution: The minimum distance between any pair of vertices. Having good vertex resolution allows an observer to quickly distinguish vertices.

Edge Length: Edges should be as short as possible, so as to allow the eye to easily identify connected vertices.

Edge Separation: Edges should be separated so as to be easily distinguished. Our formalization of this notion is a combination of two commonly used graph drawing criteria - edge resolution and angular resolution-generalized to the curved setting.

We define an offset region for each edge $e$, which defines a region around $e$ that should not contain any other edges or vertices. For each point $p$ on $e$, the offset from $p$ is defined as all points at distance at most $\delta_{e}(p)$ from $p$ along a line perpendicular to $e$ at $p$, where $\delta_{e}(p)$ is a function that depends on our drawing goals. (See Figure 1)

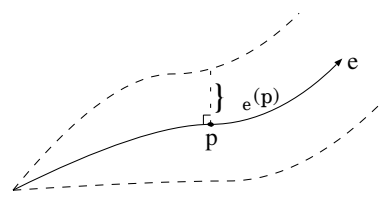

Fig. 1. The offset region of an edge $e$.

For example, if we desire an angular resolution of at least $\theta>0$ and edge resolution of at least $s>0$, then we would define

$$
\delta_{e}(p)=\min \{u \tan \theta, s\},
$$

where $u$ is the distance along $e$ from the endpoint of $e$ closer to $p$.

Thus, if $e$ is a straight line segment, then the edge separation simply states that every edge nonincident to $e$ is at least distance $s$ away and every edge incident to $e$ defines an angle of size at least $\theta$. In this typical usage, we refer to $\theta$ as the angular parameter and $s$ as the separation parameter.

Bend/Control-Point Minimization: For a polyline edge, we would like to have as few bends as possible, since many bends tend to make edges more 
difficult to follow. Analogously, as the bends in a polyline edge define the edge, so too control points define a spline or approximation curve. The more control points used to define a curve, the higher the degree of the curve or the larger the number of knots needed in a spline representation; hence, we wish to minimize the number of control points for curve edges.

Curvature Minimization: We would like edges to follow a relatively direct route between vertices, without sharp turns. The curvature of an edge is a measure of how quickly the edge bends. For polyline edges, this is simply the angle created at a bend. For curves, the curvature is measured as the rate of change of the angle of the tangent vector to the curve at any point. This, of course, can be computed directly, but a common approximation of curvature is to use the norm of the second derivative of the curve's defining equation.

These are not the only aesthetic criteria that one might wish to formalize, but they form a core set of common goals among wide classes of drawings.

\subsection{Complexity Goals}

In addition to such aesthetic criteria, there are also complexity issues for graph drawings, such as the following, which should also be considered:

Vertex Representational Complexity: Since graph drawing algorithms are typically implemented on computers, we should minimize the space needed to represent vertices. For example, many graph drawing algorithms impose the restriction that vertices be represented with integer coordinates, using $O(\log n)$ bits each. Such drawings are called grid drawings. Notice that this condition also forces vertex resolution to be at least unit distance.

Edge Representational Complexity: Similarly, we should consider the space needed to represent edges in a drawing. For example, in grid polyline or curve drawings we require that bend points and control points be represented with integer coordinates, using $O(\log n)$ bits each. In addition, by achieving good bend/control-point minimization, we are also reducing edge representational complexity.

Area: The area of a drawing is defined as the area of the smallest rectangle that encloses the drawing. We typically desire the area of a drawing to be small while not violating our aesthetic goals. Di Battista et al. [4] and Tamassia [14 summarize several known bounds on area-tradeoffs for various aesthetic properties for different types of drawings. For example, $\Theta\left(n^{2}\right)$ worst-case area is required for planar polyline grid drawings [2, 프, 10, 12].

Another interesting example of a tradeoff between the aesthetic criteria and the complexity measures is the angular resolution of the drawing. Clearly, the angular resolution of any graph drawing is at most $2 \pi / d$, where $d$ is the degree of the graph being drawn. But for a planar straight line grid drawing with $O\left(n^{2}\right)$ area, the angular resolution can be shown to be $O\left(\frac{1}{n^{2}}\right)$ (e.g., see [14]). However, allowing the edges to be polylines, Kant [10] shows how to achieve an angular resolution of $\Theta\left(\frac{1}{d}\right)$ in linear time with at most three bends per edge. 


\subsection{Characterizing Our Algorithms in This Framework}

Operating within this unified framework, we give $O(n)$ time algorithms that build on the canonical-ordering approach of the algorithms of de Fraysseix, Pach, and Pollack [3] and Kant's algorithm [10] to achieve the following results for grid drawings:

- each edge is a polygonal chain (cubic Bézier curve) with at most two bends (non-endpoint control points) per edge, such that each edge is monotonically increasing or decreasing in both $x$ and $y$ coordinates,

- vertices and bend (control) points are located at points in an $O(n) \times O(n)$ integer grid (which implies an $O(1)$ aspect ratio).

- the polyline drawing achieves an edge separation with separation parameter $s=1$ and angular parameter $\theta$ that is $\Theta(1 / d)$, where $d$ is the degree of the graph; the curvilinear drawing algorithm achieves these bounds for its control curves.

We begin the presentation of the algorithm by first reviewing the canonical ordering of the vertices of a planar graph [3, 10].

\section{Our Polyline Drawing Algorithm}

Let $G$ be an $n$-vertex plane graph of degree $d$. In addition, without loss of generality, we assume that $G$ is maximal, since we can triangulate the faces of $G$ without increasing the degree of any vertex in $G$ by more than a constant factor.

\subsection{The Canonical Ordering}

The following canonical ordering of the vertices of $G$ is due to de Fraysseix, Pach, and Pollack 3] (and extendeded by Kant [10]). Let $a, b$, and $c$ be the external vertices of $G$. A canonical ordering of the vertices of $G$ (with respect to this embedding) is a labeling $v_{1}=a, v_{2}=b, v_{3}, \ldots, v_{n}=c$ such that:

- the graph $G_{k}$ is biconnected, internally triconnected (the subgraph of $G_{k}$ induced on the interior vertices of $G_{k}$ is triconnected), and $C_{k}$ contains the edge $\left(v_{1}, v_{2}\right)$;

- vertex $v_{k+1}$ is on $C_{k+1}$ and has at least two neighbors in $G_{k}$ on the path $C_{k}-\left(v_{1}, v_{2}\right)$, all consecutive,

where $G_{k}$ is the subgraph of $G$ induced on vertices $v_{1}, v_{2}, \ldots, v_{k}$ and $C_{k}$ is the exterior face of $G_{k}$. We will refer to the vertices of $C_{k}$ in order, meaning their clockwise order on $C_{k}$ beginning with $v_{1}$. Such an ordering can be constructed in $O(n)$ time [3, 10]. 


\subsection{Our Algorithmic Approach}

Our algorithm is a blending of de Fraysseix, Pach, and Pollack's ideas [3] and the algorithm that achieves angular resolution with three bends per edge due to Kant [10. The novel aspect of our algorithm is in the development and use of a simple structure for each vertex, which we call the join box.

Let $V(G)$ be the vertex set of graph $G$, and let $d(v)$ denote the degree of vertex $v$. For each $v \in V(G)$, take a square around $v$ with corners $d(v)-1$ units above, below, left, and right of $v$. This is the join box associated with $v$ (see Figure 21). This construction is quite simple, yet it is the key to being able to reduce the number of bends per edge to two.

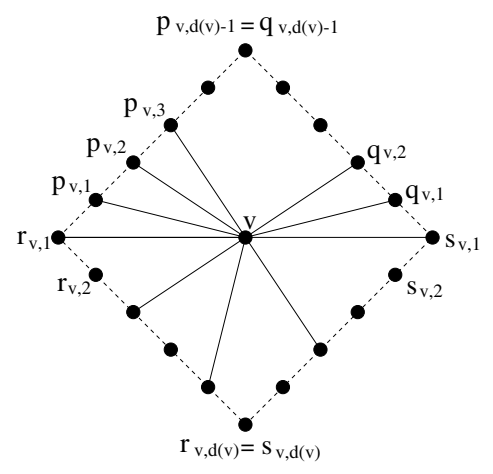

Fig. 2. A vertex $v$ 's join box.

Notice that if $v$ is located at a lattice point (integer grid point), then there are $4 d(v)-4$ lattice points located on $v$ 's join box, called join points. We label the join points as indicated in Figure 2

The algorithm adds one vertex to the drawing of $G$ during each phase so that the join points of the new vertex can connect to the join points of its neighbors via a straight line segment without any edge crossings. The join points will become the bends in the polyline edges of the graph, so that edge $(u, v)$ will have two bends, one at a join point of $u$ and the other at a join point of $v$.

The algorithm proceeds iteratively. At phase $k$ of the algorithm, the $k^{\text {th }}$ subgraph of $G, G_{k}$, has been embedded in the plane without edge crossings, while maintaining the following invariants:

1. The vertices and hence the join points of the $k^{t h}$ subgraph $G_{k}$ are located at lattice points.

2. Let $w_{1}=v_{1}, w_{2}, w_{3}, \ldots, w_{m}=v_{2}$ be the vertices of the exterior face $C_{k}$ of $G_{k}$ in order and let $x\left(w_{i}\right)$ be the $x$-coordinate of vertex $w_{i}$. Then $x\left(w_{1}\right)<$ $x\left(w_{2}\right)<\ldots<x\left(w_{m}\right)$.

3. Let $\left[w_{i}, w_{i+1}\right]$ be an edge connecting join points of $w_{i}$ and $w_{i+1}$. Then $\left[w_{i}, w_{i+1}\right]$ either has slope +1 or $-1, \forall i, 1 \leq i<m$. 
4. For each $v \in V\left(G_{k}\right)$, the join points $r_{v, 1}$ and $s_{v, 1}$ have been used and all of $v$ 's edges to its neighbors in $G_{k}$ have been drawn. Also, the upper join points that have been used are consecutive beginning at $p_{v, 1}$ and $q_{v, 1}$ (as in Figure 2).

5. Each edge is monotonically increasing (decreasing) in both the $x$ and $y$ directions.

Notice that the contour of the exterior face $C_{k}$ should appear as in Figure 3 .

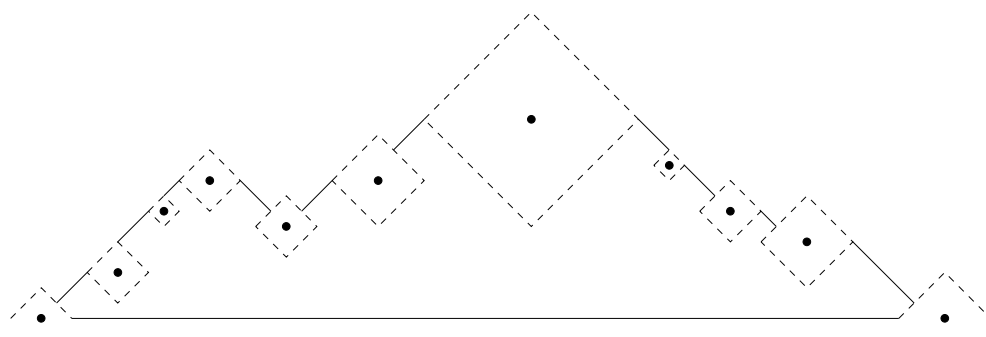

Fig. 3. Appearance of $C_{k}$ at any stage of the algorithm.

Now, consider when the algorithm is in phase $k+1$, i.e., ready to construct $G_{k+1}$ from $G_{k}$. First, note that as in the work of de Fraysseix, Pach, and Pollack [3], invariant (2) along with the fact that the sides of the join boxes have slopes of +1 and -1 imply that any two join points on the exterior face of $G_{k}$ have an even Manhattan distance 1 . This property will allow us to initially place $v_{k+1}$. If we let $p(a, b)$ be the intersection of a line with slope +1 through point $a$ and a line with slope -1 through point $b$, where $x(a)<x(b)$ then for any two join points, $a$ and $b, p(a, b)$ is a lattice point. Also, notice that invariants $(2)$ and (3) imply that the line segments from $a$ to $p(a, b)$ and from $b$ to $p(a, b)$ do not intersect $G_{k}$. So, we can add vertex $v_{k+1}$ by finding its leftmost and rightmost neighbors on $C_{k}$, choosing suitable join points, $a$ and $b$, of these neighbors, and initially placing the bottom of $v$ 's join box at $p(a, b)$. Unfortunately, this initial placement of $v_{k+1}$ does not satisfy invariant (4). This issue is resolved by carefully shifting the vertices of the drawing of the $k^{\text {th }}$ subgraph $G_{k}$ to create space for $v_{k+1}$, and then inserting $v_{k+1}$ along with edges to its neighbors on the outer face $C_{k}$, while maintaining all four invariants. Invariant (5) will be particularly useful when we replace the polyline edges by Bézier curves.

Let $w_{1}=v_{1}, w_{2}, w_{3}, \ldots, w_{m}=v_{2}$ be the vertices of the outer face $C_{k}$ of $G_{k}$ in the order of their appearance from left to right, and let $w_{l}$ and $w_{r}$ be the leftmost and rightmost neighbors, respectively, of $v_{k+1}$ on $C_{k}$. (Note that the neighbors of $v_{k+1}$ in $G_{k}$ form a contiguous sequence of vertices $w_{l}, w_{l+1}, \ldots, w_{r}$ on the exterior face $C_{k}$ of $G_{k}$.) Also, let $q_{l, i}$ be the next unused join point on the right side of $w_{l}$ 's join box and let $p_{r, j}$ be the next unused join point on the

${ }^{1}$ The Manhattan distance is the standard $l_{1}$ distance. In this case, $d(u, v)=$ $|x(u)-x(v)|+|y(u)-y(v)|$. 
left side of $w_{r}$ 's join box. We will initially place the bottom of $v_{k+1}$ 's join box be the second bend point in edge $\left(w_{o}, v_{k+1}\right)$. The remainder of the join points on the southwest side of $v_{k+1}$ 's join box will go to $w_{l+1}, \ldots, w_{o-1}$ and the join points on the southeast side of $v_{k+1}$ 's join box will go to $w_{o+1}, \ldots, w_{r-1}$. This will guarantee that edges $\left(w_{l}, v_{k+1}\right), \ldots,\left(w_{o}, v_{k+1}\right)$ are all monotonically increasing and edges $\left(w_{o+1}, v_{k+1}\right), \ldots,\left(w_{r}, v_{k+1}\right)$ are all monotonically decreasing. (We need only make sure that $v_{k+1}$ 's join points are assigned to its neighbors in such a way that there are no edge crossings. That is, if $w_{i}$ and $w_{i+1}$ are assigned join points $a$ and $b$ respectively, then $x(a)<x(b)$.) Also, notice that by shifting, since we will only increase the $x$ coordinate of any vertex $v$, and thus any vertices to the right of $v$, we do not destroy the monotonicity of the edges.

Thus, the key to the algorithm is in determining the sets of vertices to be shifted so that edge crossings cannot result from this shifting.

In the full version of this paper we show how to define shifting sets in $O(n)$ time so that shifting of vertices will not introduce any edge crossings. In fact, this argument allows us to achieve the desired edge separation. To see this, we give a brief inductive argument.

In the base case, this condition holds, since, for example, $v_{1}$ 's join box will never intersect the convex hull of edge $\left(v_{2}, v_{3}\right)$ because vertices are always shifted to the right. We can see that this condition still holds at the inductive step by noting that it holds for the darkly shaded region of Figure 4 by applying the inductive hypothesis to $G_{k}$ and by noticing that the only new edges added are incident on $v_{k+1}$ so that they all are above the darkly shaded region. Hence, $v_{k+1}$ 's join box does not intersect any nonincident edges, and no other join boxes can intersect the convex hulls of the edges incident on $v_{k+1}$. Thus, the convex hulls of nonincident edges do not intersect, implying the nonincident edges themselves do not intersect, since we will be drawing with Bézier curves.

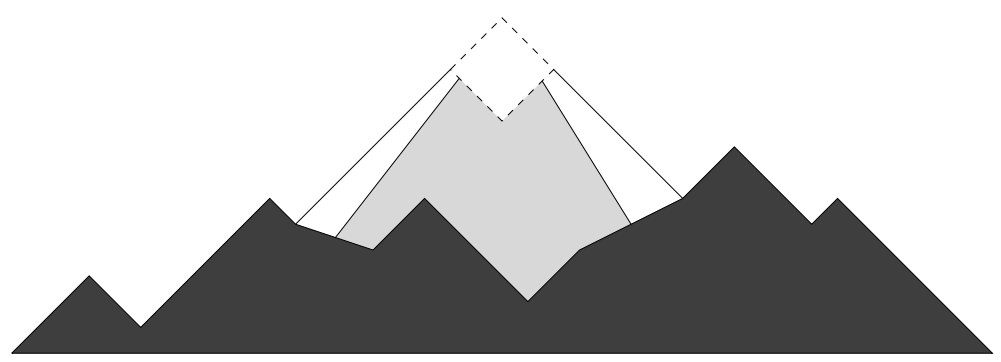

Fig. 4. The graph after shifting.

In fact, because the vertices and bend points are located at integer coordinates and because the convex hull of each edge does not intersect any nonincident join box, the convex hulls of the edges have a separation of at least unit distance, implying that the edge separation of nonincident edges is at least unit distance.

It remains to analyze the algorithm. 


\subsection{Properties of the Embedding}

First, it is clear that we have a planar polyline drawing of $G$, with at most two bends per edge. We now examine the angular resolution of the drawing of $G$ and the size of the grid on which $G$ is drawn.

Lemma 1. The size of the minimum angle is $\frac{1}{d}$, where $d$ is the maximum degree of any vertex of $G$.

Proof. Let $v \in V(G)$ have degree $d$. Then, the minimum angle, $\Theta$, occurs between a horizontal join segment, and a neighboring join segment. For $d \leq 5$, the lemma is easily verified. So, assume $d \geq 6$. The size of the angle is $\Theta=\arctan \left(\frac{1}{d-2}\right)$. By the MacLaurin series expansion of $\arctan$, we know that for $|x|<1$, $\arctan (x)=$ $x-\frac{1}{3} x^{3}+\frac{1}{5} x^{5}-\frac{1}{7} x^{7}+\ldots \geq x-\frac{1}{3} x^{3}$. Since $\frac{1}{d-2}<1$, we have $\Theta \geq \frac{1}{d-2}-\frac{1}{3}\left(\frac{1}{d-2}\right)^{3}>$ $\frac{1}{d-2}>\frac{1}{d}$, completing the proof.

Lemma 2. The size of the grid is at most $(20 n-48) \times(10 n-24)$.

Proof. Since $G$ is drawn entirely within the convex hull of vertices $\left\{v_{1}, v_{2}, v_{3}\right\}$, to find the length of the grid, we need the distance between $r_{1,1}$ and $s_{2,1}$, and to find the height, we need only find $p\left(r_{1,1}, s_{2,1}\right)$. We know that $v_{1}$ never moves, so the length is simply the distance that $v_{2}$ is shifted to the right, which is bounded by $\sum_{i=1}^{n}\left(4 d\left(v_{i}\right)-4\right)=4(6 n-12-n)=20 n-48$. But this implies that the height of the grid is $10 n-24$, and the proof is complete.

\subsection{Implementation Details and Running Time}

Chrobak and Payne 2 give a linear time implementation of the algorithm of de Fraysseix, Pach, and Pollack [3] (which originally was published as an $O(n \log n)$ time algorithm) that is easily extended to our algorithm.

The details are omitted in this extended abstract.

With this linear implementation, we arrive at the following theorem:

Theorem 1. There is a $O(n)$ time algorithm to draw a planar graph on a grid with the following properties:

- each edge is a polygonal chain with at most 2 bends per edge,

- each edge is monotonically increasing or decreasing in both its $x$ and $y$ coordinates,

- vertices and bend points are located at integer grid points,

- the size of the minimum angle created by two edges is at least $\frac{1}{d}$,

- the size of the grid is $O(n) \times O(n)$,

- there is at least unit distance between nonincident edges,

- and the drawing achieves edge separation with angular parameter $\theta=\frac{1}{d}$ and separation parameter $s=1$.

Figure 5 is a graph on 10 vertices drawn using our algorithm. 


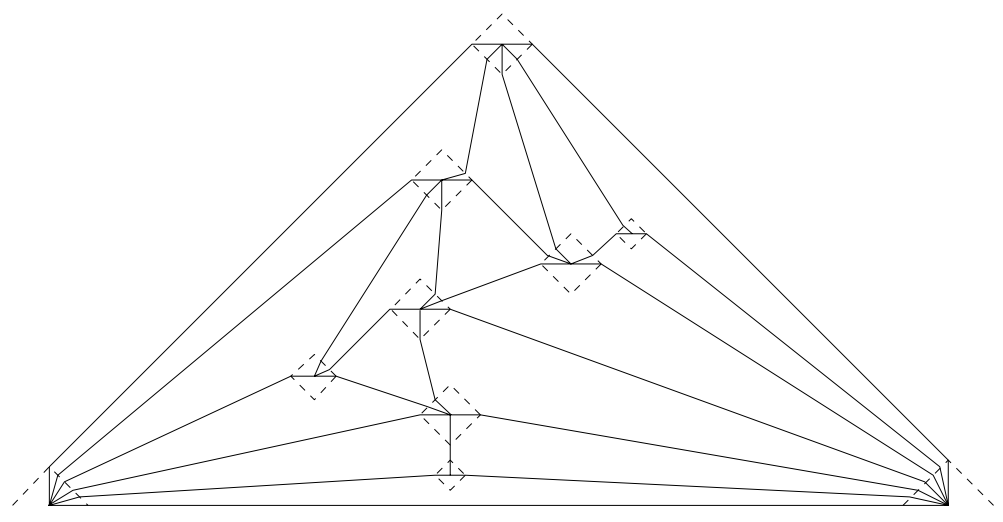

Fig. 5. A drawing of a graph on 10 vertices.

\section{Drawing with Curves}

We will now use some properties of the polyline grid drawing to show that the edges can be replaced by curves while still maintaining the desired aesthetic criteria. As mentioned, the type of approximating curve used in our algorithm is a cubic Bézier curve.

A cubic Bézier curve is defined by four control points $V_{0}, V_{1}, V_{2}$, and $V_{3}$. The curve interpolates $V_{0}$ and $V_{3}$, with the two segments $\left(V_{0}, V_{1}\right)$ and $\left(V_{2}, V_{3}\right)$ giving the tangents of the curve at the respective endpoints. The curve $C$ is defined parametrically as $C=V_{0}(1-u)^{3}+3 V_{1} u(1-u)^{2}+3 V_{2} u^{2}(1-u)+V_{3} u^{3}$, where $u \in[0,1]$. A particularly useful property of Bézier curves is that the curve stays within the convex hull of the four control points.

We will argue that if we replace each polyline edge by a Bézier curve, where the control points of the curve are the vertices and bend points of the polyline edge, we do not introduce any edge crossings. In fact, we also are able to maintain edge separation (angular and edge resolution), as defined in the framework.

We deal with two cases, the case of nonincident edges and the case of incident edges. We have already argued that a join box does not intersect the convex hull of a nonincident edge, implying that we have unit separation of nonincident edges.

We now address the case of incident edges. Since the tangents of each curve simply correspond to the first segments of the polyline edges, we have angular resolution of $\frac{1}{d}$ in the planar curve grid drawing. Before arguing that incident edges do not cross, recall that all edges are monotonically increasing or decreasing. Consider edge $e$. Without loss of generality, the control points of $e$ are $(0,0),\left(x_{1}, y_{1}\right),\left(x_{2}, y_{2}\right)$, and $\left(x_{3}, y_{3}\right)$, with $0 \leq x_{1} \leq x_{2} \leq x_{3}$ and $0 \leq y_{1} \leq y_{2} \leq y_{3}$. Consider another edge incident at $(0,0)$, call it $f$. We want to examine the separation between $e$ and $f$. When measuring edge separation, we want to consider the minimum distance between any pair of incident edges. Clearly, this distance would be achieved by parallel edges, if they were allowed 
in the graph. So, we will consider the case of parallel edges and show that we have a measure of edge resolution for parallel edges, implying that we have that same edge separation for incident edges.

So, to make $e$ and $f$ as close as possible, let the control points of $f$ be $(0,0),\left(x_{1}+1, y_{1}-1\right),\left(x_{2}+1, y_{2}-1\right)$, and $\left(x_{3}, y_{3}\right)$, as in Figure 6.

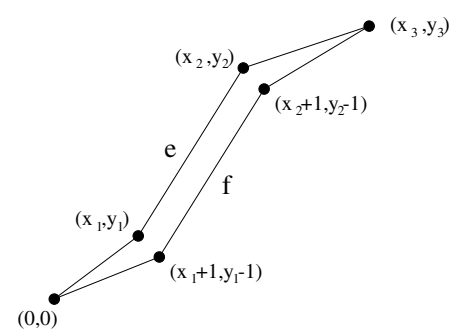

Fig. 6. Parallel edges $e$ and $f$.

The $x$ and $y$ coordinates of edge $e$ are given by $x_{e}=3 u(1-u)^{2} x_{1}+3 u^{2}(1-$ $u) x_{2}+u^{3} x_{3}$ and $y_{e}=3 u(1-u)^{2} y_{1}+3 u^{2}(1-u) y_{2}+u^{3} y_{3}$, respectively. Similarly, the $x$ and $y$ coordinates of edge $f$ are given by $x_{f}=3 u(1-u)^{2}\left(x_{1}+1\right)+3 u^{2}(1-$ $u)\left(x_{2}+1\right)+u^{3} x_{3}$ and $y_{f}=3 u(1-u)^{2}\left(y_{1}-1\right)+3 u^{2}(1-u)\left(y_{2}-1\right)+u^{3} y_{3}$, respectively. Taking differences, we have $x_{f}-x_{e}=3 u(1-u)$ and $y_{f}-y_{e}=$ $-3 u(1-u)$. This means that for any $u^{*} \in[0,1]$, we have a point $e\left(u^{*}\right)$ on $e$, and the corresponding point on $f, f\left(u^{*}\right)$, is $3 u(1-u)$ units below and to the right of $e\left(u^{*}\right)$. For convenience, this is how we opt to initially measure the edge resolution of incident edges; ie, the distance between edges along a line of slope \pm 1 . So, the minimum distance between incident edges is at most $3 u(1-u) / \sqrt{2}$. (We deal with monotonically increasing edges here, but the argument for decreasing edges is identical.)

This fact, coupled with the monotonicity of the polyline edges implies that converting the polyline edges to cubic Bézier curves does not introduce edge crossings. To see this, suppose that $e$ and $f$ cross. Then there is a point on $f$ that is above some point on $e$. Call these points $f^{*}$ and $e^{*}$, respectively. Then $x\left(f^{*}\right)=x\left(e^{*}\right)$ and $y\left(f^{*}\right)>y\left(e^{*}\right)$. Let $\hat{u}$ be the parameter value that gives this point $f^{*}$. Then, $e(\hat{u})$ is above and to the right of $f^{*}$. But if this is the case, then $e$ must come back down from $e(\hat{u})$ to pass through the point $e^{*}$, contradicting the monotonicity of the edges.

See Figure 7 for an example of a curve drawing.

This gives the following theorem:

Theorem 2. There is a $O(n)$ time algorithm to draw a planar graph on a grid with the following properties:

- each edge is a cubic Bézier curve,

- each edge is monotonically increasing or decreasing in both its $x$ and $y$ coordinates, 


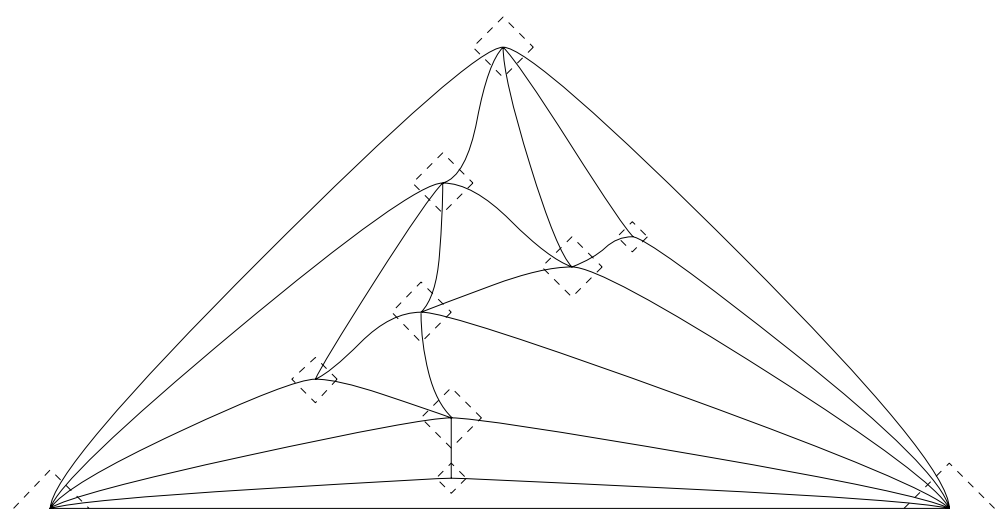

Fig. 7. The graph from Figure 5 drawn with curve edges.

- vertices and control points are located at integer grid points,

- the size of the minimum angle created by two incident edges is at least $\frac{1}{d}$, as measured by the tangents of the curves,

- the size of the grid is $O(n) \times O(n)$,

- there is at least unit distance between nonincident edges,

- and the drawing has angular parameter $\theta=\frac{1}{d}$ and separation parameter $s=1$ for the control curves.

\section{Comments and Open Problems}

Our algorithm can easily be extended to triconnected graphs, just as Kant 10 extends the algorithm of de Fraysseix, Pach, and Pollack. When we initially place a chain of vertices, we simply locate the initial point based on the vertex of maximum degree in the chain. Then, we shift by the sum of the degrees of the vertices in the chain.

Having established a framework for drawing graphs with curves, there are any number of aesthetic criteria that can be redefined for curve drawings, leading to some directions of further research:

- Devise new algorithms to draw graphs with curves, operating under this general framework.

- Devise a dynamic algorithm for drawing graphs with curve edges.

- If one of the control points of the curve edges is far from the remaining three, the curvature of the edge can be quite large, in fact proportional to $n$. One may be able to devise an algorithm that also minimizes the curvature of the edges.

- Because there are roughly $4 d(v)$ join points on $v$ 's join box and we only use $d(v)$ of them, we waste a great deal of space. There may be a way of cleverly reducing the sizes of the join boxes and thus the constants involved in the area of the drawing. 
- Since we use the upper join points in order, often the join points near the top of the join box are not used. There may be some heuristics that allow for a better "spacing" of the upper join points once the final layout is constructed.

\section{References}

[1] T. Chan, M. T. Goodrich, S. R. Kosaraju, and R. Tamassia. Optimizing area and aspect ratio in straight-line orthogonal tree drawings. In S. North, editor, Graph Drawing (Proc. GD '96), volume 1190 of Lecture Notes Comput. Sci., pages 63-75. Springer-Verlag, 1997.

[2] M. Chrobak and T. Payne. A linear-time algorithm for drawing planar graphs. Inform. Process. Lett., 54:241-246, 1995.

[3] H. de Fraysseix, J. Pach, and R. Pollack. How to draw a planar graph on a grid. Combinatorica, 10:41-51, 1990.

[4] G. Di Battista, P. Eades, R. Tamassia, and I. G. Tollis. Algorithms for drawing graphs: an annotated bibliography. Comput. Geom. Theory Appl., 4:235-282, 1994.

[5] M. Formann, T. Hagerup, J. Haralambides, M. Kaufmann, F. T. Leighton, A. Simvonis, E. Welzl, and G. Woeginger. Drawing graphs in the plane with high resolution. SIAM J. Comput., 22:1035-1052, 1993.

[6] E. R. Gansner, E. Koutsofios, S. C. North, and K. P. Vo. A technique for drawing directed graphs. IEEE Trans. Softw. Eng., 19:214-230, 1993.

[7] A. Garg and R. Tamassia. Planar drawings and angular resolution: Algorithms and bounds. In Proc. 2nd Annu. European Sympos. Algorithms, volume 855 of Lecture Notes Comput. Sci., pages 12-23. Springer-Verlag, 1994.

[8] J. Hopcroft and R. E. Tarjan. Efficient planarity testing. J. ACM, 21(4):549-568, 1974.

[9] G. Kant. Algorithms for Drawing Planar Graphs. PhD thesis, Dept. Comput. Sci., Univ. Utrecht, Utrecht, Netherlands, 1993.

[10] G. Kant. Drawing planar graphs using the canonical ordering. Algorithmica, 16:4-32, 1996. (special issue on Graph Drawing, edited by G. Di Battista and R. Tamassia).

[11] S. Malitz and A. Papakostas. On the angular resolution of planar graphs. SIAM J. Discrete Math., 7:172-183, 1994.

[12] W. Schnyder. Embedding planar graphs on the grid. In Proc. 1st ACM-SIAM Sympos. Discrete Algorithms, pages 138-148, 1990.

[13] R. Tamassia. On embedding a graph in the grid with the minimum number of bends. SIAM J. Comput., 16(3):421-444, 1987.

[14] R. Tamassia. Graph drawing. In J. E. Goodman and J. O'Rourke, editors, Handbook of Discrete and Computational Geometry, chapter 44, pages 815-832. CRC Press LLC, Boca Raton, FL, 1997. 